\title{
Emerging Liquid Crystal Displays Based on the Kerr Effect
}

\author{
LINGHUI RAO, ZHIBING GE, SEBASTIAN GAUZA, \\ KUAN-MING CHEN, AND SHIN-TSON WU
}

College of Optics and Photonics, University of Central Florida, Orlando, Florida, USA

\begin{abstract}
The device physics and electro-optical properties of emerging liquid crystal displays ( $L C D s)$ based on the Kerr effect are investigated. With a voltage applied, both blue phase and polymer-stabilized blue phase exhibit a fascinating isotropic-toanisotropic transition. We have developed a numerical model to understand the underlying device physics in order to optimize device performance. The operating voltage is found to be inversely proportional to the square-root of Kerr constant of the LC composite. To reduce operation voltage while keeping high transmittance and fast response time, device structures with strong and deep horizontal electric fields, and LC materials with a large Kerr constant play equally important roles.
\end{abstract}

Keywords Blue phase liquid crystals; fast response time; Kerr effect

\section{Introduction}

Liquid crystal displays (LCDs) have been widely used in mobile phones, video games, navigational devices, laptop computers, desktop monitors, TVs, and data projectors [1]. After about three decades of extensive development effort, and heavy investment in manufacturing technology, it seems as though LCD technology has reached mature stage. The most critical issue - viewing angle - has been overcome by using multidomain structures [2-4] and optical-film compensation [5,6]. The next most frequently mentioned shortcoming - response time - has been improved to $2-5 \mathrm{~ms}$ or less through low-viscosity LC material development, overdrive and undershoot voltage methodology [7], and thin cell-gap approach [8,9]. Motion picture image blur has been significantly reduced by using impulse driving, frame insertion, and fast-response liquid crystals [10]. The color shift at off-axis viewing directions has been dramatically reduced by using an eight-domain approach via two transistors [11-13]. The contrast ratio has exceeded 1,000,000:1 through LED-backlight local dimming [14,15]. The color gamut would exceed 120\% NTSC if RGB LEDs were used [16]. Even with all these technological advances, the cost has also been reduced dramatically through investment in advanced cost-effective manufacturing facilities.

Address correspondence to Shin-Tson Wu, College of Optics and Photonics, University of Central Florida, Orlando, FL 32816, USA. E-mail: swu@mail.ucf.edu 
Recently, a promising new technology involving blue-phase liquid crystal (BPLC) $[17,18]$ is emerging. BPLC exists in a very narrow temperature range (1$2{ }^{\circ} \mathrm{C}$ ) between the chiral-nematic and isotropic phases; its molecular structure is made up of double-twist cylinders arranged in a cubic lattice with periods of $\sim 100 \mathrm{~nm}$ $[19,20]$. BPLCs have been explored for several decades; however, their mesogenic temperature range was always too narrow for practical applications. But recently this situation changed when a small amount of polymer was embedded to stabilize the LC lattice structure [21-23]. The polymer-stabilized blue phase showed a reasonably wide mesogenic temperature range, covering room temperature. At the voltage-off state, BPLC appears optically isotropic. As the voltage increases, the induced birefringence increases based on the Kerr effect and the LC refractive-index distribution becomes anisotropic. When the device is sandwiched between two crossed polarizers, the transmittance gradually increases as the voltage increases.

In comparison to conventional nematic LCDs, polymer-stabilized blue phase exhibits four revolutionary features:

1. It does not require any alignment layer, such as polyimide, which not only simplifies the manufacturing processes but also reduces the cost.

2. Its response time is in the submillisecond range, which helps to minimize the motion-image blur and, more importantly, enables color-sequential displays without pigment color filters if an RGB LED backlight is used. The major impacts of eliminating color filters are threefold: (i) it enhances optical efficiency by $\sim 3 \mathrm{X}$, resulting in lower power consumption if the same display brightness is compared; (ii) it increases device resolution by $3 \mathrm{X}$ (i.e., crisper images); and (iii) it reduces production cost.

3. The dark state of a blue-phase LCD is optically isotropic so that its viewing angle is wide and symmetric. Optical compensation films may or may not be needed, depending on the actual applications.

4. The transmittance is insensitive to the cell gap, as long as the cell gap exceeds $2-3 \mu \mathrm{m}$ depending on the birefringence of the LC composite employed. This cellgap insensitivity is particularly attractive for fabricating large-screen or singlesubstrate LCDs [24], in which cell-gap uniformity is a big concern.

Although polymer-stabilized blue-phase LCDs hold so much promise, some tough technical issues remain to be overcome before widespread applications can take off. The major challenges are in three aspects: (1) The operation voltage is still too high $\left(\sim 50 \mathrm{~V}_{\mathrm{rms}} v s .5 \mathrm{~V}_{\mathrm{rms}}\right.$ for conventional nematic LCDs), (2) the transmittance is relatively low ( $\sim 65 \%$ vs. $85 \%$ for nematic LCDs), and (3) the mesogenic temperature range is still not wide enough for practical display applications (from $-40^{\circ} \mathrm{C}$ to $80^{\circ} \mathrm{C}$ ). The operating voltage of a blue-phase LCD is primarily governed by the induced birefringence which in turn is dependent on the Kerr constant $(K)$ of the LC composite and the electric-field strength. Therefore, developing new blue-phase LC materials with a large Kerr constant and new device structures for enhancing the horizontal electric-field intensity in the in-plane-switching electrode configuration are urgently needed.

In this paper, we discuss the device operation mechanisms of LCDs based on the Kerr effect. These general discussions apply to any Kerr effect-induced isotropicto-anisotropic transition, including blue phase and polymer-stabilized blue-phase LCDs. Our objective is to lower the operation voltage while keeping high transmittance and fast response time. 


\section{Device Operation Mechanism}

Kerr effect is a type of quadratic electro-optic effect caused by an electric-fieldinduced ordering of polar molecules in an optically isotropic medium. It usually exists in crystals with centro-symmetric point groups. Refractive index change $(\delta n)$ is induced by the external electric field $(E)$, and its magnitude is proportional to the square of the electric field as $[25,26]$ :

$$
\delta n=\lambda K E^{2}
$$

Here, $\lambda$ is the wavelength, $K$ is the Kerr constant, and $E$ is the amplitude of the electric field. In Eq. (1), the induced refractive index change is linearly proportional to $E^{2}$ in the low field regime and will gradually saturate as $E$ increases, but its maximum value cannot exceed the effective birefringence of the LC composite. From the Kerr effect, the LC composite is optically isotropic when there is no voltage applied, but will become anisotropic when the electric field is present.

It has been discovered that the Kerr effect is present also in blue phase liquid crystals. Blue phase is a type of liquid crystal phase that has a molecular structure comprising of double-twist cylinders arranged in a cubic lattice with periods of $\sim 100 \mathrm{~nm}$. Recently, the polymer stabilization method has successfully widened the temperature range of BPLC to over 60 degrees including room temperature [20]. The coexistence with disclination lines stabilizes such self-assembled three-dimensional periodic structures.

As the temperature increases, there are three thermodynamically stable blue phases, BP-I, BP-II, and BP-III. BP-I and BP-II exhibit three-dimensional periodic structures in the director field. They have body-centered and simple cubic symmetry, respectively. BP-III is seemingly amorphous with a cubic local structure [17]. As the electric field intensity increases, there will be three distinct transformations in BPLCs: 1) local reorientation of molecules caused by Kerr effect: its response time is about $0.1 \mathrm{~ms}$; 2) lattice distortion in BP-I: its response time is about $10 \mathrm{~ms}$; and 3) phase transition to a lower-symmetry phase, i.e., switching from BP-I to BP-II: its response time is more than a few seconds [27]. Therefore, the submillisecond-response LCDs based on Kerr effect enable color sequential displays using a RGB LED backlight unit [28].

\section{Numerical Model}

Our group has developed a 3-D numerical model to calculate the electro-optic properties of blue-phase LCDs [29,30], or more generally speaking, a device involves isotropic-to-anisotropic transition based on Kerr effect. Here we briefly outline our approaches as follows. We first solve the Poisson equation $\nabla(\nabla \cdot \varepsilon \Phi)=0$ to obtain the potential distribution $(\Phi)$ and the electric field distribution $(E)$ in the LC media. We then calculate the induced birefringence by Eq. (1), limit it to be below the effective birefringence of the $\mathrm{LC} /$ polymer composite and assign the local optic axis direction of each unit to be along the $E$ vector. Finally, we characterize the voltage-dependent transmittance and other electro-optic properties with extended Jones matrix. The modeling results are discussed as follows. 


\subsection{Wavelength Effect}

The following single-band model describes the wavelength dependent Kerr constant $[31,32]$ :

$$
\lambda K \approx G \frac{\lambda^{2} \lambda^{* 2}}{\lambda^{2}-\lambda^{* 2}},
$$

here $G$ is a proportionality constant and $\lambda^{*}$ the mean resonance wavelength. In this model, we used $\mathrm{G} \sim 8.78 \times 10^{-2} \mathrm{~V}^{-2}$ and $\mathrm{K} \sim 12.68 \mathrm{~nm} / \mathrm{V}^{2}$ at $\lambda=550 \mathrm{~nm}$. For display applications, we need to know the wavelength-dependent Kerr constant at other primary wavelengths. Let us assume the employed LC mixture has a similar birefringence dispersion as E-7 whose $\Delta \mathrm{n} \sim 0.22$ and $\lambda^{*} \sim 250 \mathrm{~nm}$ due to elongated $\pi$-electron conjugation. We then calculate by Eq. (2) the Kerr constants for $\lambda=450 \mathrm{~nm}$ and $650 \mathrm{~nm}$ to be $\mathrm{K} \sim 17.6 \mathrm{~nm} / \mathrm{V}^{2}$ and $\sim 9.9 \mathrm{~nm} / \mathrm{V}^{2}$, respectively. Figures 1(a) and 1(b) depict the simulated voltage-dependent transmittance (VT) curves of the blue-phase and nematic LC cells, respectively, with in-plane-switching (IPS) electrodes; the electrode width is $w=5 \mu \mathrm{m}$ and spacing $l=10 \mu \mathrm{m}$. The cell gap for the blue phase $\mathrm{LC}$ is $10 \mu \mathrm{m}$ and $3.5 \mu \mathrm{m}$ for the nematic.

From Figure 1, the color dispersion of BPLC for red, green, and blue is larger than that of the nematic. In a conventional nematic IPS cell, self-compensation effect exists for wavelength dispersion because the on-state LC profile acts like two connected reversely twisted nematic cells [33]. However, in an IPS BPLC cell, the transmittance originates from the pure phase retardation of the Kerr effect; it does not have such a self-compensation. The shorter wavelength has a larger Kerr constant, so a lower driving voltage results. Meanwhile, this pure phase retardation from the Kerr effect also leads to a uniform peak transmittance for each wavelength in BPLC, which is different from the nematic IPS cell.

\subsection{Electrode Effect}

Electrode structure affects the driving voltage and the peak transmittance of an IPS BPLC cell. The induced birefringence of BPLC is proportional to $\mathrm{E}^{2}$, and is influenced by both amplitude and direction of the electric field. Since the induced LC ellipsoid is along the electric field direction, a horizontal electric field is needed to produce phase retardation for intensity modulation. Thus, in an IPS cell the transmittance is mainly gained in the spacing area where the electric field is more horizontally distributed. There is only a tiny and negligible contribution on top of the pixel and common electrodes where the electric field is mostly in vertical direction.

The simulation results shown in Figure 2 are all with $K=12.68 \mathrm{~nm} / \mathrm{V}^{2}$. First, let us compare the three $10-\mu \mathrm{m}$ IPS cells with electrode width $w=2 \mu \mathrm{m}$ and spacing $l$ varying from $2 \mu \mathrm{m}$ to $4 \mu \mathrm{m}$ (open squares, triangles, and circles). Both on-state voltage and peak transmittance decrease as the electrode spacing decreases. For the same electrode width, a smaller electrode spacing $l$ results in a stronger horizontal electric field, so the driving voltage is lower. However, the smaller electrode spacing reduces the effective area of the horizontal field from which the transmittance is gained, so the optical efficiency is also lower. We further compare the 10- $\mu \mathrm{m}$ IPS cell with electrode width $w=5 \mu \mathrm{m}$ and spacing $l=10 \mu \mathrm{m}$ (IPS 5-10, blue filled circles) with the $10-\mu \mathrm{m}$ IPS cell with electrode width $w=2 \mu \mathrm{m}$ and spacing $l=4 \mu \mathrm{m}$ (IPS 2-4, magenta 


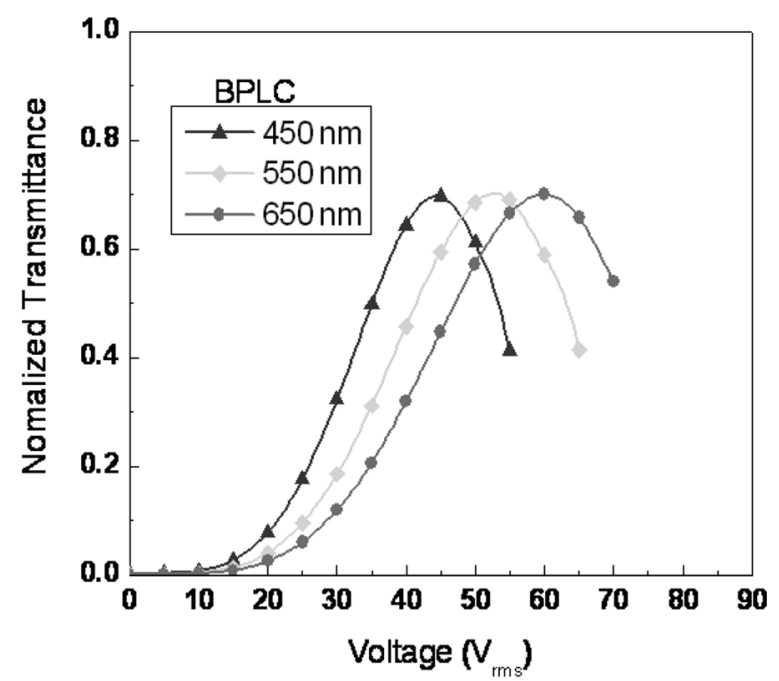

(a)

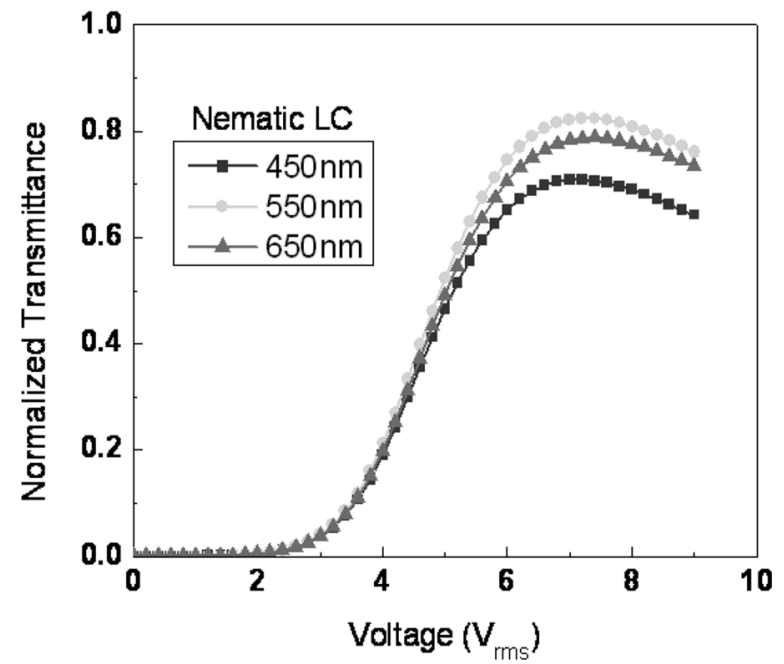

(b)

Figure 1. Voltage-dependent transmittance curves of IPS cells with $5-\mu \mathrm{m}$ electrode width and $10-\mu \mathrm{m}$ electrode spacing at R $(650 \mathrm{~nm}), \mathrm{G}(550 \mathrm{~nm})$, and B $(450 \mathrm{~nm})$. (a) BPLC: cell gap $\mathrm{d}=10 \mu \mathrm{m}$; (b) nematic LC: cell gap $\mathrm{d}=3.5 \mu \mathrm{m}, \Delta \mathrm{n}=0.085$.

open circles). They have the same $w / l$ ratio. Again, the lower driving voltage results from a stronger electric field due to a smaller electrode spacing $(l=4 \mu \mathrm{m})$. IPS 5-10 has a slightly higher peak transmittance than IPS 2-4. This can be explained by the depth of the electric field penetrating from the bottom electrodes to the LC layer. According to the Poisson equation of $\nabla^{2} \Phi=0$, the penetrating depth is proportional to the dimensional size $(w+l)$. Thus, the electric field penetrates deeper into the LC layer in the IPS 5-10 cell than IPS 2-4, resulting in a higher induced birefringence and then peak transmittance. As described earlier, the area on top of the electrodes in BPLC cells do not contribute to the transmittance because the electric field is 


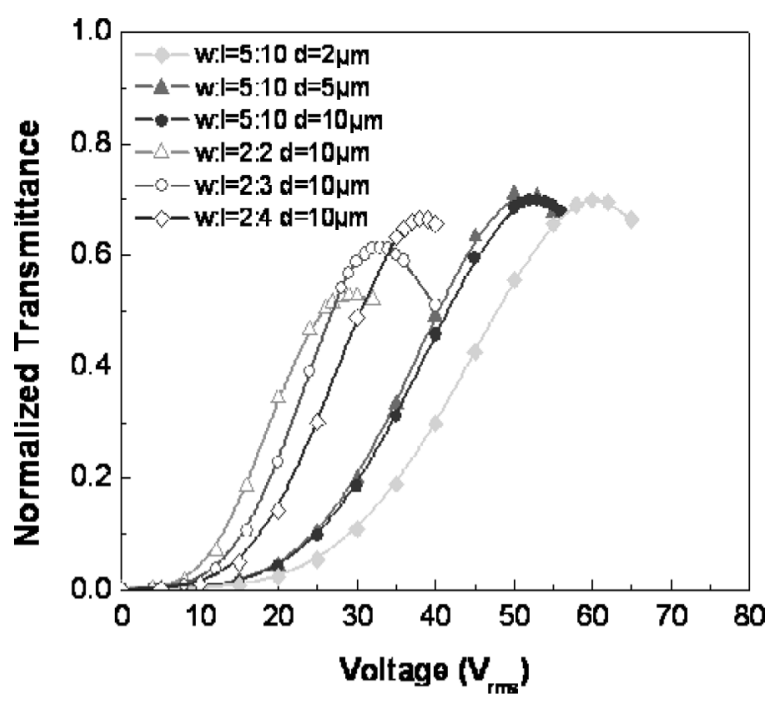

Figure 2. Voltage-dependent transmittance of BP IPS cells with different $w: l$ ratios and cell gaps.

primarily longitudinal. However the conventional nematic IPS cell, unlike the BPLC, has a weak in-plane LC rotation above the electrodes that enhance the optical efficiency. As a result, the overall optical transmittance of the BPLC cells could be lower than that of conventional IPS nematic cells.

\subsection{Induced Birefringence Distribution and Cell Gap Effect}

The transmittance of a nematic IPS cell is largely affected by the cell gap, since the phase is continuously accumulated along the cell gap. However, in an IPS BPLC cell the transmittance is quite insensitive to the cell gap. As illustrated in Figure 2, for the electrode dimensions $w=5 \mu \mathrm{m}$ and $l=10 \mu \mathrm{m}$ (IPS 5-10), the transmittance remains the same for cell gap $d=5 \mu \mathrm{m}$ and $10 \mu \mathrm{m}$.

To better understand the transmittance and electric field distribution, we plot the induced birefringence distribution at on-state voltage (where maximum transmittance occurs) in Figures 3 and 4 for IPS 5-10 and IPS 2-4, respectively. Two different Kerr constants are employed for comparison: $K_{a}=1.268 \mathrm{~nm} / \mathrm{V}^{2}$ and $K_{b}=12.68 \mathrm{~nm} / \mathrm{V}^{2}$. Here $\delta n$ denotes the overall induced birefringence by the applied electric field, regardless of the field direction; $\delta n_{\text {eff }}$ is the effective birefringence for the normal incident light that contributes to the phase change. For IPS 5-10, the on-state voltage is $\mathrm{V}_{\text {on }}=167 \mathrm{~V}_{\text {rms }}$ at $K_{a}$ and $53 \mathrm{~V}_{\text {rms }}$ at $K_{b}$; while for IPS 2-4, $\mathrm{V}_{\text {on }}=121 \mathrm{~V}_{\text {rms }}$ at $K_{a}$ and $38 \mathrm{~V}_{\text {rms }}$ at $K_{b}$. We can see from the plot that with the same electrode dimension, the Kerr constants do not affect the on-state induced birefringence.

From Figures 3(a), 3(c), 4(a), and 4(c), on top of the electrodes, although $\delta n$ is comparatively large and extended deep into the LC layer, it is induced by the vertical electric field which does not contribute to the transmittance. This is also verified that in Figures 3(b), 3(d), 4(b), and 4(d) where $\delta n_{\text {eff }}$ is almost zero on top of the electrodes. The induced birefringence at the spacing area is not only very small but also 

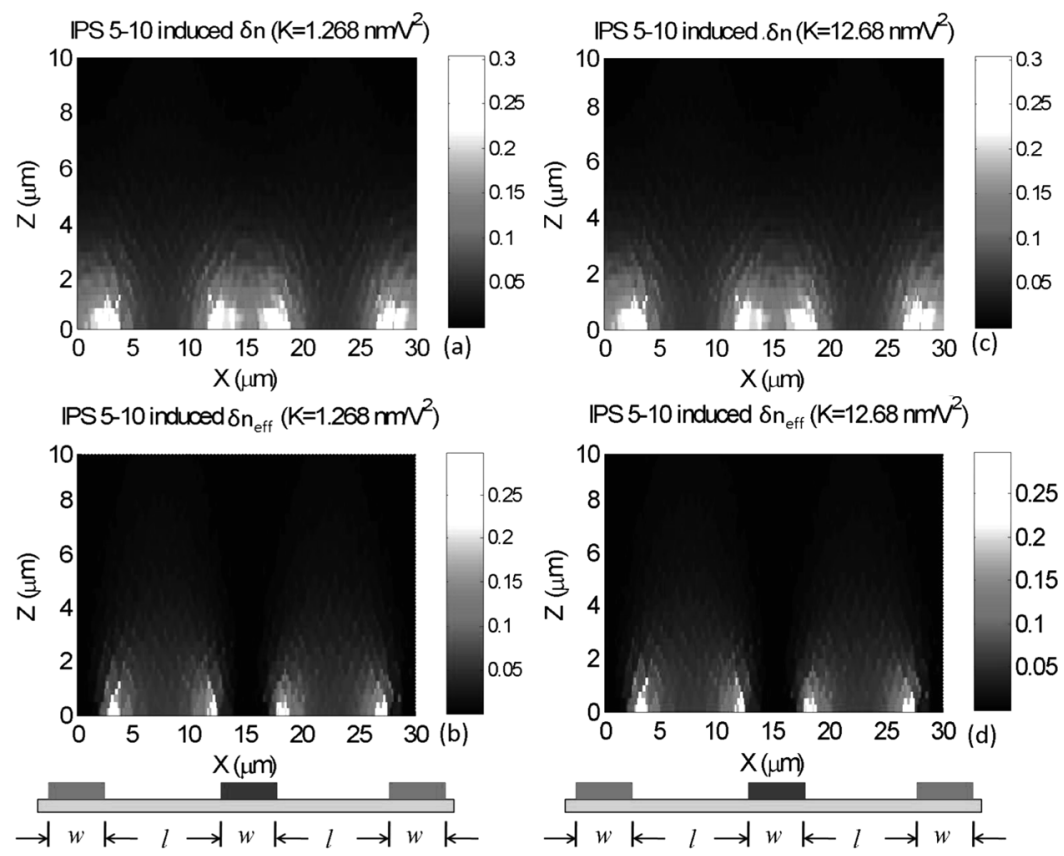

Figure 3. Spatial profiles of induced birefringence and effective birefringence in IPS cells: $w$ is the electrode width, $l$ is the spacing width. (a) overall induced $\delta n$ of IPS 5-10 at $K_{a}$, (b) induced $\delta n_{\text {eff }}$ of IPS 5-10 at $K_{a}$, (c) overall induced $\delta n$ of IPS 5-10 at $K_{b}$, (d) induced $\delta n_{\text {eff }}$ of IPS 5-10 at $K_{b} .\left(K_{b}=10 K_{a}=12.68 \mathrm{~nm} / \mathrm{V}^{2}\right)$.

decays fast with the cell gap. Thus, as long as the cell gap is larger than the fairly small penetrating depth in the vertical direction, the transmittance will be insensitive to the cell gap. The tiny difference between the $5-\mu \mathrm{m}$ and $10-\mu \mathrm{m}$ cells of IPS $5-10$ structure in Figure 2 may come from the passivation layer. It has a much smaller dielectric constant than the LC composite, making the electric energy more concentrated in the LC layer of the 5- $\mu \mathrm{m}$ cell. If the cell gap is smaller than the effective penetrating thickness, for example, when the IPS 5-10 has a cell gap of $d=2 \mu \mathrm{m}$, we find in Figure 2 that the on-state voltage is higher. In this case, a larger electric field is needed to gain the same phase change in a smaller cell gap. As shown in Figures 3 and 4, IPS 5-10 and IPS 2-4 differ in that the latter has a shorter penetrating depth, a faster decay in birefringence, and its amplitude near the electrode bottom is larger. This is actually the result of the electric field concentration difference, and again it explains why IPS 2-4 yields a lower driving voltage and a slightly lower transmittance.

\subsection{Kerr Constant Effect on Driving Voltage}

The operating voltage of a blue-phase LCD is primarily governed by the induced birefringence which in turn is dependent on the Kerr constant of the LCs and the electric field strength. On one hand, investigation on the device structure unveils influence from the electric field to the driving voltage and the transmittance; on the other hand, from the material side the LC parameters directly affect the display 

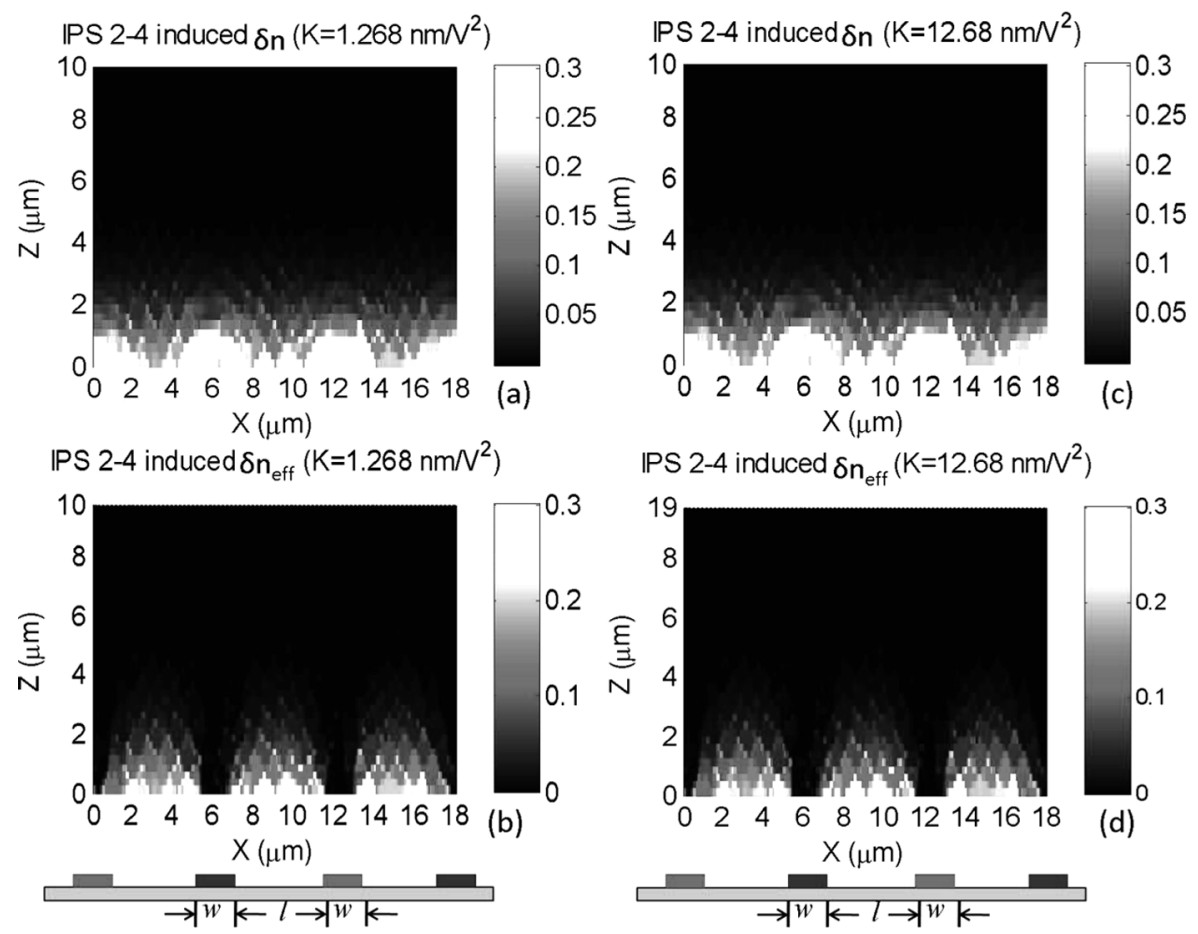

Figure 4. Spatial profiles of induced birefringence and effective birefringence in IPS cells: $w$ is the electrode width, $l$ is the spacing width. (a) overall induced $\delta n$ of IPS 2-4 at $K_{a}$, (b) induced $\delta n_{\text {eff }}$ of IPS 2-4 at $K_{a}$, (c) overall induced $\delta n$ of IPS 2-4 at $K_{b}$, (d) induced $\delta n_{\text {eff }}$ of IPS 2-4 at $K_{b}$. $\left(K_{b}=10 K_{a}=12.68 \mathrm{~nm} / \mathrm{V}^{2}\right)$.

performance. In this section, one of the most important factors, the Kerr constant, is analyzed.

Figures 5(a) and 5(b) are the plots of simulated VT curves for $K_{1}=12.68 \mathrm{~nm} / \mathrm{V}^{2}$, $K_{2}=2 K_{1}, K_{3}=5 K_{1}$ and $K_{4}=10 K_{1}$ for two IPS structures: $w=2 \mu \mathrm{m}, l=4 \mu \mathrm{m}$, and $w=5 \mu \mathrm{m}, l=10 \mu \mathrm{m}$. For both structures, the peak transmittance does not change with $K$. Figure 5(c) depicts the on-state voltages for the above four $K$ values as well as $K_{5}=0.1 K_{1}$ and $K_{6}=0.2 K_{1}$. Surprisingly, the on-state voltages exhibit a linear relationship with $K^{-1 / 2}$, although the electric field is quite nonuniform. The red and blue lines are the linear fittings with the following equation:

$$
V_{o n}=A \cdot \frac{1}{\sqrt{K}}
$$

The proportionality constant $A$ for IPS $2-4$ and IPS $5-10$ is $A_{1}=4.31 \mu \mathrm{m}^{1 / 2}$ and $A_{2}=5.94 \mu \mathrm{m}^{1 / 2}$, respectively. With the same Kerr constant, the on-state voltage of IPS $2-4$ is $\sim 40 \%$ lower than that of IPS $5-10$, but its transmittance is slightly $(\sim 3 \%)$ lower. For low power TFT-LCDs, it is highly desirable to have $\mathrm{V}_{\text {on }}<10 \mathrm{~V}_{\text {rms. }}$. To achieve this goal, from Figure 5 we need to continue to improve the Kerr constant of the BPLC material and optimize the electrode configuration.

As referred to Figure 3, although the $K$ value may not be the same, the induced birefringence distribution at its corresponding on-state voltage is basically the same, 

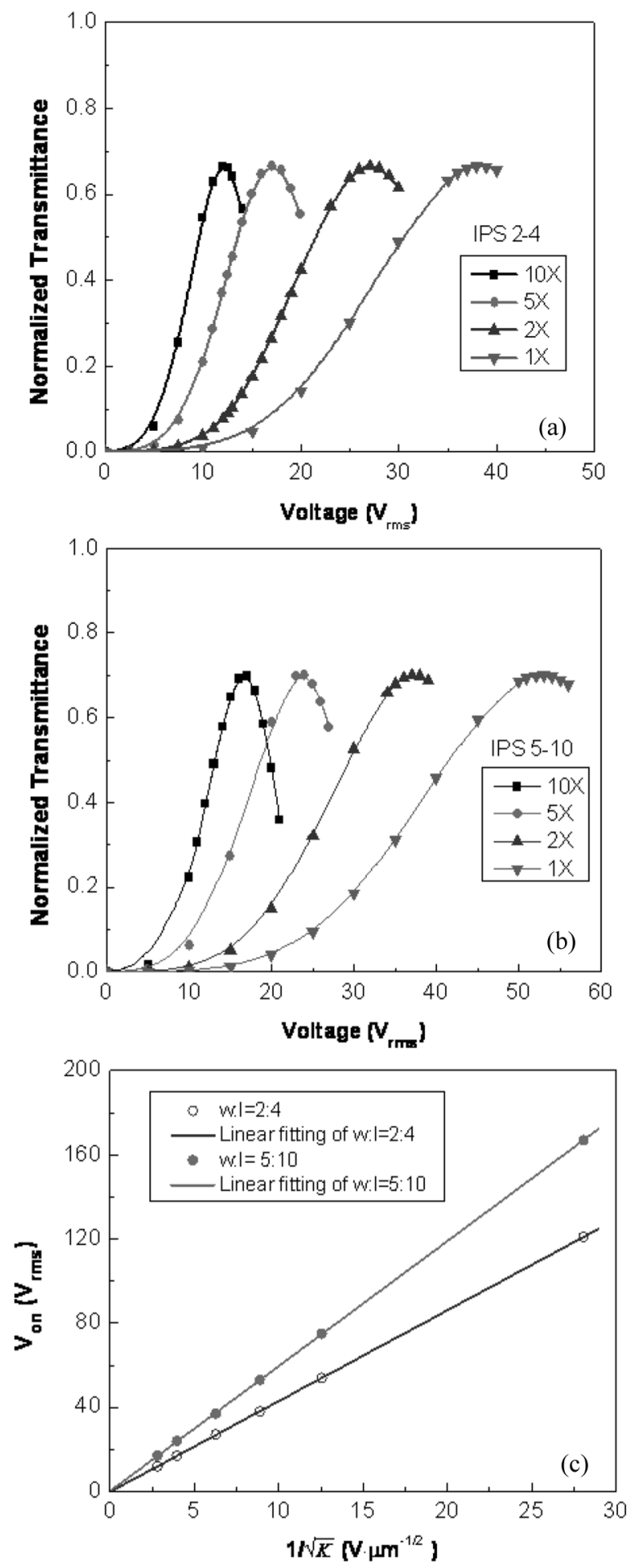

Figure 5. (a) Voltage-dependent transmittance curves with different Kerr constants for IPS 2-4, (b) V-T curves with different Kerr constants for IPS 2-4, (c) linear plot between on-state voltage and $1 / \sqrt{K}$. 
and thus results in a same peak transmittance. According to Eq. (1), at a given wavelength the induced birefringence is directly related to $K E^{2}$. Then, we can conclude that for a given IPS electrode dimension operating at the on-state voltage, if the electric field $E$ is uniform in the IPS cell, then $K E^{2}$ will be a constant. As $E=\mathrm{V} / \mathrm{L}, \mathrm{KV}^{2}$ is also a constant, and Eq. (3) holds for this straightforward case. However, in a real IPS structure, $E$ is not uniform spatially. It is surprising to see that the linear relationship as shown in Eq. (3) is still valid according to our simulation results. In an IPS cell, the $K E^{2}$ value may differ at different area because of the $E$ distribution, but it remains a constant for the same area in the cell regardless of the variance of $K$. The voltage $V$ is related to the electric field $E$ as $E=\partial V / \partial L$ ( $\partial L$ is the infinitesimal change in distance), as long as the cell is operated under the on-state voltage, the $K V^{2}$ value would stay constant for the same area of the cell despite of the $K$ variation.

\subsection{FFS vs. IPS}

Current research of BPLC for display applications is usually conducted under the traditional strip electrode structure using IPS cells. In this section, we discuss
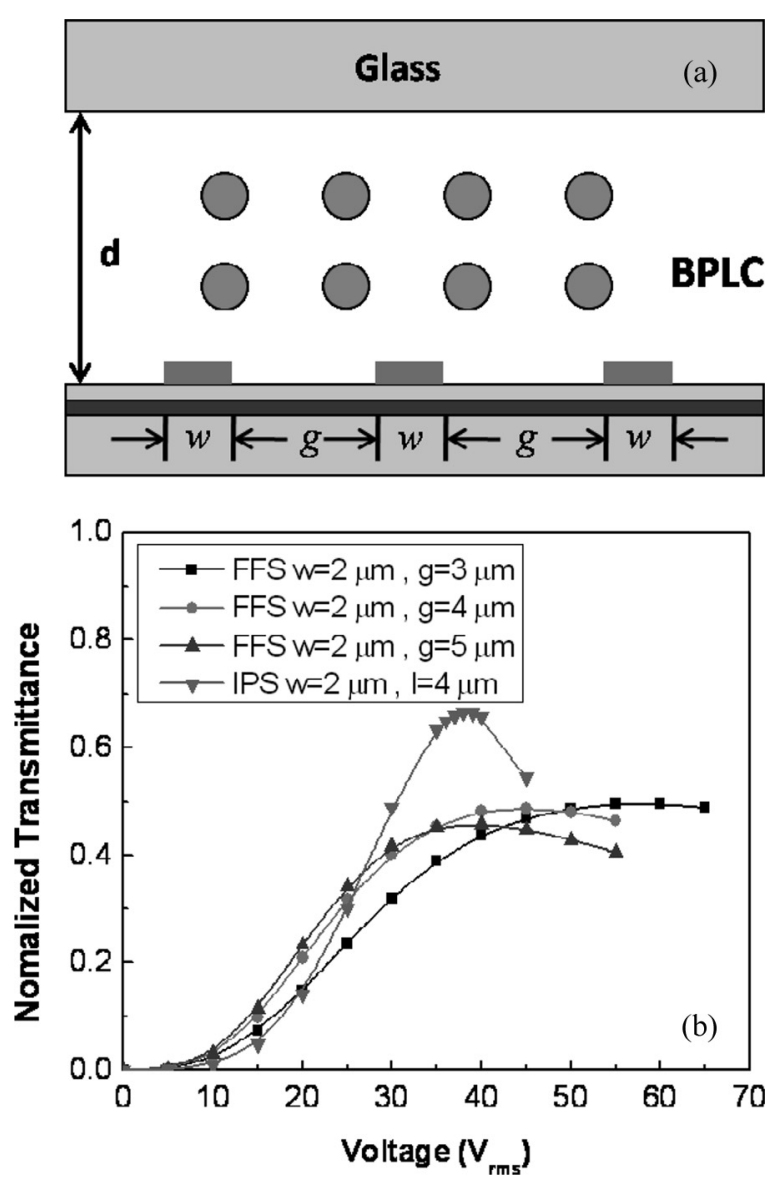

Figure 6. (a) Voltage-dependent transmittance curves for IPS and FFS cells with different electrode dimensions. Cell gap is $10 \mu \mathrm{m}$ for all the curves, (b) an FFS cell structure. 
fringing field switching (FFS) structure [34], which is also widely employed in wide-view nematic LCDs. Electrode dimension in a FFS cell is distinguished by the electrode width $w$ and the gap distance $g$ between two electrodes (Fig. 6(a)). Both $w$ and $g$ are smaller than the cell gap. If we compare the FFS structure with different $w: g$ ratios under the same cell gap $\mathrm{d}=10 \mu \mathrm{m}$, curves in Figure 6(b) demonstrate the trend that the smaller the $w: g$ ratio, the lower the on-state voltage and the faster the transmittance increases at a low voltage. This is because with the same electrode width $w$, a larger gap $g$ provides a deeper penetration of the electric field in the LC layer which helps to lower the operation voltage.

In Figure 6(b), IPS BPLC has much higher optical efficiency at the on-state voltage than the FFS structure. IPS provides more horizontal electric field in the spacing area, while FFS structure exerts a strong vertical field. In the FFS cell, the vertical field is in favor to the reorientation of nematic LCs on top of the electrodes for enhancing transmittance, but does not contribute to the birefringence we need in BPLCs. However, at low operating voltages, such as between $10-25 \mathrm{~V}_{\mathrm{rms}}$, the transmittance of the FFS structure is higher and increases faster than that of the IPS structure. The strongest horizontal electric field is present at the edges of the electrodes but decreases to the minimum at the center of the gap center between two electrodes. From the potential distribution in a FFS cell, the electric field at electrode edge is much stronger than that in an IPS cell. It can quickly rotate the LCs, so that the transmittance in FFS rises faster than that of IPS cell in the low voltage regime. As the voltage increases, the horizontal field in IPS cell becomes stronger than FFS cell so that FFS cannot compete with IPS cell for the peak transmittance. Therefore, FFS offers a steeper transmittance in low voltage but saturates at a much lower transmittance than IPS, as shown in Figure 6(b). This phenomenon has been confirmed in our experiment (data not shown here).

\section{Discussion}

Developing new blue phase LC materials with a large Kerr constant is extremely important for reducing the driving voltage. As shown in Figure 5, the on-state voltage is dramatically reduced with the increased Kerr constant. It has been reported that the Kerr constant can be approximated by the following equation [35]:

$$
K \sim \frac{\delta n}{\lambda E^{2}} \approx \Delta n \cdot \Delta \varepsilon \frac{\varepsilon_{o} P^{2}}{k \lambda(2 \pi)^{2}}
$$

Here, $\delta n$ is the induced birefringence, $\lambda$ is the wavelength, $E$ is the electric field; $\Delta n$, $\Delta \varepsilon$ and $k$ are the intrinsic birefringence, dielectric anisotropy, and elastic constant of the host LC material. $P$ is the pitch length that influences the Bragg reflection wavelength $\lambda_{B}$ as [36]:

$$
\lambda_{B}=\langle n\rangle P,
$$

where $\langle n\rangle$ is the average refractive index of the LC. From Eq. (4), to enhance the Kerr constant, the host LC composite with a high birefringence, large dielectric anisotropy, long pitch length, and small elastic constant is preferred. Especially, increasing pitch length has a quadratic effect on the Kerr constant. In a normal 
BPLC, we design $P$ to have Bragg reflection at UV so that it will not affect the display performance in the visible region. If we shift $\lambda_{B}$ to near infrared by increasing $P$, then the higher order Bragg reflection could occur in the visible region. For displays, any reflection band in the visible range should be avoided. Another big concern for increasing pitch length is the slower response. The response time of a blue phase LC can be approximated by [36]:

$$
\tau \approx \frac{\gamma_{1} P^{2}}{k(2 \pi)^{2}}
$$

with $\gamma_{1}$ being the rotational viscosity, and $k$ the elastic constant.

According to Eqs. (4) and (6), controversy exists also in the elastic constant $k$ : a small $k$ enlarges the Kerr constant but increases the response time. However, high intrinsic birefringence, large dielectric anisotropy, and low viscosity are always in favor. Therefore, to better evaluate the performance of a BPLC material, a delicate balance between each parameter, such as operating voltage, transmittance and response time, should be taken into consideration [37-40].

\section{Conclusion}

Liquid crystal displays based on the Kerr effect have demonstrated attractive features in simple fabrication process and fast response time. Symmetric and wide viewing angles, as well as low color shift can also be achieved. To overcome the existing high operating voltage, we have explored the device physics based on the Kerr effect and investigated on the electro-optical properties. Developing new blue phase LC materials with a large Kerr constant and new device structures for enhancing the horizontal electric field intensity and penetrating deeper into the LC layer are urgent tasks for the emergence of blue phase display technology. Once the existing high voltage problem is properly addressed, the LCD technology will be advanced to a new level.

\section{Acknowledgments}

The authors are indebted to Chi-Mei Optoelectronics (Taiwan) for partial financial support.

\section{References}

[1] Yang, D. K., \& Wu, S. T. (2006). Fundamentals of Liquid Crystal Devices, Wiley: New York.

[2] Takeda, A., Kataoka, S., Sasaki, T., Chida, H., Tsuda, H., Ohmuro, K., Sasabayashi, T., Koike, Y., \& Okamoto, K. (1998). SID Symposium Digest, 29, 1077.

[3] Oh-e, M., \& Kondo, K. (1995). Appl. Phys. Lett., 67, 3895.

[4] Kim, H., Song, J., Park, S., Lyu, J., Souk, J., \& Lee, K. (2000). J. Soc. Info. Displays, 1, 3.

[5] Hong, Q., Wu, T. X., Zhu, X., Lu, R., \& Wu, S. T. (2005). Appl. Phys. Lett., 86, 121107.

[6] Zhu, X., Ge, Z., \& Wu, S. T. (2006). J. Display Technol., 2, 2.

[7] Wu, S. T., \& Wu, C. S. (1989). J. Appl. Phys., 65, 527.

[8] Wu, S. T., \& Efron, U. (1986). Appl. Phys. Lett., 48, 624.

[9] Jiao, M., Ge, Z., Song, Q., \& Wu, S. T. (2008). Appl. Phys. Lett., 92, 061102. 
[10] Hong, H., Shin, H., \& Chung, I. (2007). J. Display Technol., 3, 361.

[11] Kim, S. S. (2005). SID Symposium Digest, 36, 1842.

[12] Liu, C. T. (2007). J. Display Technol., 3, 342.

[13] Lu, R., Wu, S. T., \& Lee, S. H. (2008). Appl. Phys. Lett., 92, 051114.

[14] de Greef, P., \& Hulze, H. G. (2007). SID Symposium Digest, 38, 1332.

[15] Peng, H. J., Zhang, W., Hung, C.-K., Tsai, C.-J., Ng, K.-W., Chen, S., Huang, D., Chueng, Y.-L., \& Liu, Y. (2007). SID Symposium Digest, 38, 1336.

[16] Lu, R., Hong, Q., Ge, Z., \& Wu, S. T. (2006). Opt. Express, 14, 6243.

[17] Crooker, P. P. (2001). In: Chirality in Liquid Crystals, Kitzerow, H. S. \& Bahr, C. (Ed.), Springer: New York.

[18] de Gennes, P. G., \& Prost, J. (1993). The Physics of Liquid Crystals, 2nd Ed., Clarendon: Oxford.

[19] Meiboom, S., Sethna, J. P., Anderson, W. P., \& Brinkman, W. F. (1981). Phys. Rev. Lett., 46, 1216.

[20] Memmer, R. (2000). Liq. Cryst., 27, 533.

[21] Kikuchi, H., Yokota, M., Hiskado, Y., Yang, H., \& Kajiyama, T. (2002). Nat. Mater., 1,64 .

[22] Haseba, Y., Kikuchi, H., Nagamura, T., \& Kajiyama, T. (2005). Adv. Mater., 17, 2311.

[23] Hisakado, Y., Kikuchi, H., Nagamura, T., and Kajiyama, T. (2005). Adv. Mater., 17, 96.

[24] Lin, Y. H., Ren, H., Gauza, S., Wu, Y. H., Zhao, Y., Fang, J., \& Wu, S. T. (2006). J. Display Technol., 2, 21.

[25] Yan, J., Cheng, H. C., Gauza, S., Li, Y., Jiao, M., Rao, L., \& Wu, S. T. (2010). Appl. Phys. Lett., 96, 071105.

[26] Yan, J., Jiao, M., Rao, L., \& Wu, S. T. (2010). Opt. Express, 18, 11450.

[27] Sato, M., \& Yoshizawa, A. (2007). Adv. Mater., 19, 4145.

[28] Gauza, S., Zhu, X., Piecek, W., Dabrowski, R., \& Wu, S. T. (2007). J. Display Technol., $3,250$.

[29] Ge, Z., Gauza, S., Jiao, M., Xianyu, H., \& Wu, S. T. (2009). Appl. Phys. Lett., 94, 101104.

[30] Ge, Z., Rao, L., Gauza, S., \& Wu, S. T. (2009). J. Display Technol., 5, 250.

[31] Wu, S. T. (1986). Phys. Rev. A., 33, 1270.

[32] Wu, S. T., Wu, C. S., Warenghem, M., \& Ismaili, M. (1993). Opt. Eng., 32, 1775.

[33] Ge, Z., Wu, S. T., Kim, S. S., Park, J. W., \& Lee, S. H. (2008). Appl. Phy. Lett., 92, 181109.

[34] Lee, S. H., Lee, S. L., \& Kim, H. Y. (1998). Appl. Phys. Lett., 73, 2881.

[35] Gerber, P. R. (1985). Mol. Cryst. Liq. Cryst., 116, 197.

[36] Wu, S. T., \& Yang, D. K. (2001). Reflective Liquid Crystal Displays, New York: Wiley.

[37] Rao, L., Ge, Z., Wu, S. T., \& Lee, S. H. (2009). Appl. Phys. Lett., 95, 231101.

[38] Jiao, M., Li, Y., \& Wu, S. T. (2010). Appl. Phys. Lett., 96, 011102.

[39] Chen, K. M., Gauza, S., Xianyu, H., \& Wu, S. T. (2010). J. Display Technol., 6, 49.

[40] Rao, L., Ge, Z., \& Wu, S. T. (2010). J. Display Technol., 6, 115. 